\title{
Perspective
}

PERSPECTIVE Actualité en histoire de l'art

$2 \mid 2011$

Les Pays-Bas

\section{L'architecture médiévale aux Pays-Bas : vingt-cinq années de recherches et de problématiques}

Medieval Netherlandish architecture: twenty-five years of research

\section{Thomas Coomans et Jeroen Westerman}

\section{(2) OpenEdition}

1 Journals

Édition électronique

URL : http://journals.openedition.org/perspective/776

DOI : $10.4000 /$ perspective. 776

ISSN : 2269-7721

Éditeur

Institut national d'histoire de l'art

Édition imprimée

Date de publication : 31 décembre 2011

Pagination : 783-791

ISSN : 1777-7852

\section{Référence électronique}

Thomas Coomans et Jeroen Westerman, «L'architecture médiévale aux Pays-Bas : vingt-cinq années de recherches et de problématiques », Perspective [En ligne], 2 | 2011, mis en ligne le 30 juin 2013, consulté le 01 octobre 2020. URL : http://journals.openedition.org/perspective/776 ; DOI : https:// doi.org/10.4000/perspective.776 


\section{L'architecture médiévale aux Pays-Bas : vingt-cinq années de recherches et de problématiques}

\section{Thomas Coomans et Jeroen Westerman}

En 2007 parut Bouwen in Nederland 600-2000, une nouvelle histoire de l'architecture aux Pays-Bas, du haut Moyen Âge à la fin du $\mathrm{XX}^{\mathrm{e}}$ siècle $^{1}$, qui vient bouleverser celle présentée dans les synthèses précédentes, datées de 1928-1941 et de 1957-1958, produites dans un contexte historiographique très différent ${ }^{2}$. Impliquant vingt-cinq auteurs, elle n'est plus une histoire des styles et des architectes, mais développe une approche complexe des typologies architecturales et de leurs significations culturelles et historiques (fig. 1). S'adressant à un large public, l'ouvrage est un exemple de vulgarisation scientifique, abondamment illustré et pourvu d'une bibliographie et d'index. L'architecture médiévale, de 600 à 1500, en occupe environ un tiers, alors qu'elle ne constitue qu'une infime partie du bâti aux Pays-Bas et se limite aux typologies ecclésiales et, dans une moindre mesure, aux typologies castrale et domestique urbaine.

Force est de constater, cependant, que la part consacrée au Moyen Âge au sein des publications scientifiques est de plus en plus modeste. On en veut pour preuve le faible nombre d'articles sur le sujet publié dans le Bulletin van de Koninklijke Nederlandse Oudheidkundige Bond (Bulletin $K N O B)$, la revue d'histoire de l'architecture aux Pays-Bas depuis 1899.
Ce n'est pourtant pas surprenant dans un pays où le Siècle d'or domine en raison de la quantité de bâtiments conservés, de leur état de conservation, y compris de leurs intérieurs, et de la richesse de leurs archives. L'architecture antérieure au $\mathrm{XI}^{\mathrm{e}}$ siècle, en comparaison, y est quasi inexistante. Ce n'est qu'à partir du $\mathrm{XV}^{\mathrm{e}}$ siècle que les bâtiments et les archives augmentent en volume et en signification. Enfin, comme partout ailleurs, les $\mathrm{XIX}^{\mathrm{e}}$ et $\mathrm{XX}^{\mathrm{e}}$ siècles exercent aujourd'hui sur les chercheurs une plus grande force d'attraction que le Moyen Âge.

Malgré la taille relativement modeste de ce champ de recherche, il continue à s'enrichir progressivement. Les études sur l'architecture médiévale aux Pays-Bas, en particulier l'architecture romane du $\mathrm{XI}^{\mathrm{e}}$ au début du XIII ${ }^{\mathrm{e}}$ siècle, se sont renouvelées dans les années 1980 et ont atteint un haut niveau dans les années 1990 grâce à une approche interdisciplinaire et transnationale. Depuis une dizaine d'années, ce mouvement s'est ralenti, tandis que l'archéologie du bâti, également développée dans les années 1990, a maintenu son niveau de production. Malgré la crise dans les secteurs de la recherche universitaire et de la conservation du patrimoine, un intérêt renouvelé pour l'architecture du Moyen Âge tardif se manifeste ces dernières années.

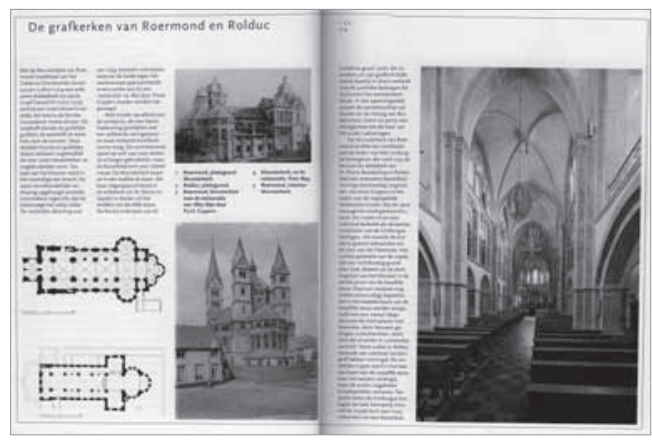

1. Notice sur « De grafkerken van Roermond en Rolduc », dans Koos Bosma et al., Bouwen in Nederland 600-2000, Amsterdam/ Zwolle, 2007, p. 146-147. 


\section{L'héritage historiographique en question}

Plusieurs générations d'historiens néerlandais de l'architecture médiévale ont porté la discipline à un haut niveau scientifique après la Seconde Guerre mondiale - notamment M. D. Ozinga, E. H. ter Kuile, R. Meischke, H. J. Zantkuijl, H. Janse, C. L. Temminck Groll -, sans toutefois parvenir à en assurer une diffusion internationale. Les raisons principales sont l'obstacle de la langue et les perspectives historiographiques essentiellement nationales. La frontière entre les Pays-Bas et la Belgique semble longtemps avoir été une barrière mentale infranchissable pour les historiens attachés à leur patrimoine national. Malgré une courte "réunification " de 1815 à 1830, les deux pays ont suivi des destinées distinctes depuis les guerres de religion de la seconde moitié du XVI ${ }^{\mathrm{e}}$ siècle et ont développé des identités et des traditions différentes. Aussi, le Bas Moyen Âge et le début de la Période moderne forment-ils la seule longue période d'unité entre les Pays-Bas septentrionaux (Pays-Bas actuels) et méridionaux (Belgique, Luxembourg et Nord de la France actuels), de 1433 à 1586, sous le règne des ducs de Bourgogne et des Habsbourg, de Philippe le Beau à Charles Quint. En raison d'une historiographie nationaliste particulièrement marquée par des identités religieuses différentes et par l'indépendance belge de 1830, les historiens ont longtemps été incapables d'envisager le Moyen Âge dans ses frontières historiques. En outre, la césure conventionnelle entre Moyen Âge et Renaissance autour de la date arbitraire de 1500 a accentué la fragmentation dans le temps et dans l'espace d'une période historique qui était cohérente.

Ce schéma de pensée s'est modifié dans les années 1980 lorsque les médiévistes

2. Planche de comparaison de tours d'églises paroissiales, dans A. J. J. Mekking, Het spel met toren en kapel: bouwen pro en contra Bourgondië van Groningen tot Maastricht, Utrecht/Zutphen, 1992, p. 18-19. néerlandais ont progressivement ouvert ces frontières historiographiques au profit d'une vision transnationale et interdisciplinaire. Le réseau interuniversitaire d'études médiévales (Onderzoekschool Mediëvistiek), fondé en 1994, a amplifié le mouvement. Il regroupe des unités de recherche des deux universités d'Amsterdam, de celles d'Utrecht, de Leyde, de Nimègue et de Groningue, ainsi que des médiévistes belges. Depuis bientôt vingt ans, ce réseau joue un rôle essentiel dans le renouvellement des problématiques, la formation des jeunes chercheurs, le développement de programmes communs et l'internationalisation de la recherche. Les travaux historiques sur la société urbaine et marchande aux $\mathrm{XV}^{\mathrm{e}}$ et $\mathrm{XVI}^{\mathrm{e}}$ siècles développés par Wim Blockmans ont définitivement orienté la recherche vers une vision globale des anciens Pays-Bas et sa mise en perspective dans le nord de l'Europe ${ }^{3}$. Il existe d'autres réseaux plus spécialisés de médiévistes aux Pays-Bas, mais ceuxci ne font pas de l'architecture une priorité ${ }^{4}$.

Parallèlement, à partir de la fin des années 1980, plusieurs historiens de l'architecture ont publié des ouvrages sur les églises romanes de Maastricht et sur l'architecture des $\mathrm{XI}^{\mathrm{e}}$ et $\mathrm{XII}^{\mathrm{e}}$ siècles dans la vallée de la Meuse ${ }^{5}$. Non seulement leur approche était transfrontalière, mais elle renouvelait également la problématique en substituant à l'histoire des styles et des écoles régionales des nouvelles perspectives sur la signification symbolique des édifices et sur le rôle de leurs commanditaires. Parmi ces auteurs, Aart Mekking, inspiré par la méthode iconologique de l'architecture telle que l'avaient définie Günter Bandmann et Richard Krautheimer ${ }^{6}$, a développé une approche holistique et iconologique de l'architecture médiévale, en remettant notamment en question les frontières héritées de l'historiographie nationaliste des XIX $^{\text {e }}$ et $\mathrm{XX}^{\mathrm{e}}$ siècles (fig. 2$)^{7}$. Initiateur et directeur du réseau "Kunst en Regio " (Art et Région) de 1995 à 2000, le professeur Mekking a formé une génération de chercheurs néerlandais, auxquels se sont associés de nombreux historiens de l'architecture allemands et quelques spécialistes belges ${ }^{8}$. De cette courte mais intense

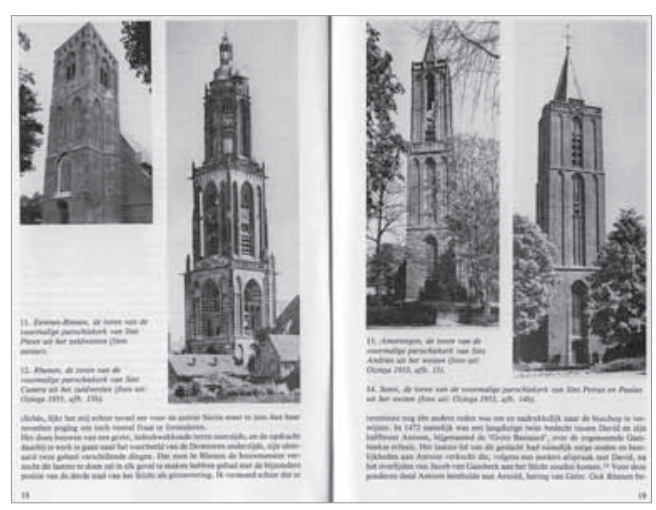


collaboration ont résulté notamment des publications doctorales et postdoctorales sur les églises urbaines du XIV siècle, l'église SaintSauveur d'Utrecht, l'église abbatiale de Rolduc, l'église d'Oldenzaal, la cathédrale de Tournai, l'architecture monastique et les représentations iconographiques d'architecture avant 1300, ainsi que deux ouvrages de mélanges ${ }^{9}$.

Après 2000, cet élan s'est ralenti et personne n'a véritablement repris le flambeau de Mekking. Seule Elizabeth den Hartog a continué de publier sur des aspects de l'architecture et de l'art romans aux Pays-Bas, respectivement sur les églises dans le comté de Hollande avant 1300 et sur les chapiteaux romans de Maastricht ${ }^{10}$. Paradoxalement, la grande architecture gothique qui se développa dans les anciens PaysBas à partir de la fin du XIV ${ }^{\mathrm{e}}$ siècle a bien moins bénéficié du renouvellement historiographique des années 1990. Sans doute le poids des études anciennes sur le "gothique brabançon " constituait-il un obstacle particulier ${ }^{11}$; ce n'est qu'en 2009 que parut une nouvelle synthèse sur le sujet ${ }^{12}$. La thèse de doctorat défendue par Merlijn Hurx en 2010 sur le développement du rôle de l'architecte sur les chantiers des anciens Pays-Bas au Moyen Âge tardif est sans aucun doute la recherche fondamentale la plus prometteuse ${ }^{13}$. Elle bénéficie à la fois du mouvement international qui cherche à aborder la " transition " entre gothique et Renaissance autrement qu'en termes de rupture ${ }^{14}$, et de la masse d'informations recueillies depuis des décennies par les archéologues du bâti.

Une autre difficulté propre à l'étude de l'architecture médiévale aux Pays-Bas est la pauvreté des intérieurs originaux, mise en évidence par les spécialistes néerlandais Justin Kroesen et Regnerus Steensma dans leur ouvrage sur les intérieurs d'églises paroissiales médiévales en Europe $^{15}$. L'iconoclasme calviniste des années 1580 a été particulièrement virulent et a détruit quasiment tous les vitraux, autels, jubés, sculptures, tombeaux et autres meubles d'église, à de rares exceptions près ${ }^{16}$. Contrairement à d'autres régions où une approche intégrée de l'architecture, de son mobilier et de son usage permet de renouveler l'historiographie, pour les Pays-Bas, les chercheurs doivent la plupart du temps se contenter de peintures murales médiévales fortuitement mises au jour sous des blanchiments successifs ${ }^{17}$. Le professeur Jos Koldeweij, spécialiste de l'art religieux et profane du Moyen Âge tardif, étudie notamment les sculptures de miséricordes des stalles, dont quelques exemplaires remarquables sont conservés aux Pays-Bas ${ }^{18}$.

\section{L'archéologie du bâti : une longue tradition} Une particularité des Pays-Bas est l'absence de matériaux de construction naturels, ce qui impliqua, dès le Moyen Âge, l'importation massive de bois d'œuvre et de pierres à bâtir ainsi que la fabrication de matériaux artificiels comme la brique ou la chaux à base de coquillages. Aussi, les historiens de l'architecture néerlandais ont-ils développé une longue tradition d'archéologie du bâti dont résulte une connaissance approfondie des matériaux et des techniques de construction. Cette expertise spécifique est également liée aux problèmes de restauration. Aux Pays-Bas, les archéologues du bâti - qui ne sont pas que des médiévistes, loin s'en faut-sont regroupés depuis 1991 en une fondation (Stichting Bouwhistorie Nederland), publient abondamment et disposent d'excellents manuels de méthode ${ }^{19}$. En 2000, la fondation a créé une chaire d'enseignement à l'université de Leyde, occupée par le professeur Dirk Jan de Vries, qui complète les différentes filières universitaires et techniques. La connaissance accumulée et publiée grâce à ce réseau professionnel bien organisé est considérable ${ }^{20}$. Elle ne se limite pas aux grands monuments ni au Moyen Âge, et porte autant d'intérêt aux maisons urbaines qu'à l'habitat rural, traditionnel et moderne.

L'étude des formes est évidemment indissociable de celle des matériaux. Sur ce terrain, les Pays-Bas se trouvent à la croisée des chemins et d'influences multiples. L'identification de l'origine et de l'époque d'utilisation des matériaux pierreux a permis aux historiens de reconstituer les circuits économiques, le transport maritime et fluvial, etc., sans oublier les marques de tâcherons et les autres signes lapidaires ${ }^{21}$. Plusieurs publications récentes d'approches très différentes, méritent d'être signalées. D’une part, l'ouvrage de Wim Dubelaar sur les pierres à Utrecht aborde, à l'échelle d'une ville, tous les aspects liés au 
3. Edwin D. Orsel, "Hausbau in Leiden", dans Gabri van Tussenbroek éd., Hausbau in Holland: baugeschichte und stadtentwicklung, Jahrbuch für Hausforschung, 61), Marburg, 2010, p. 248-249.

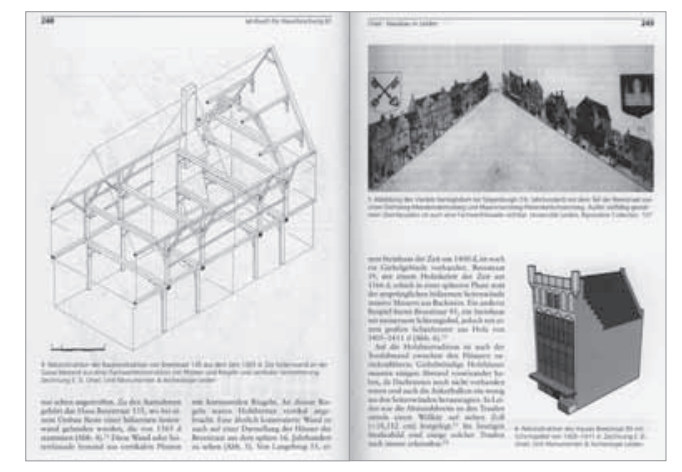

matériau à travers les siècles (provenance, taille et outils, finition, etc. ${ }^{22}$. D'autre part, les thèses de doctorat de Gabri van Tussenbroek et de Merlijn Hurx, consacrées au développement du secteur du bâtiment en fonction de la préfabrication et du commerce de la pierre de taille, portent respectivement sur les Van Neurenberg, une dynastie mosane de marchands de pierre active de 1480 à 1640, et sur les processus créatifs liés à la pierre brabançonne et l'émergence d'une génération $\mathrm{d}^{\prime}$ architectes au $\mathrm{XV}^{\mathrm{e}}$ siècle ${ }^{23}$.

Pour le bois d'œuvre provenant des pays baltes, de Scandinavie, des Ardennes et de l'Eiffel, les apports de la dendrochronologie pratiquée aux Pays-Bas depuis bientôt deux décennies ont permis d'affiner considérablement l'étude des charpentes et des structures. Grâce au travail de longue haleine et aux nombreux articles de Dirk Jan de Vries ${ }^{24}$, ainsi qu'aux analyses réalisées par le laboratoire RING depuis 1993, la datation dendrochronologique des charpentes s'est systématisée aux Pays-Bas. Il manque toutefois une nouvelle synthèse qui compléterait celle de Herman Janse de 1989, essentiellement typologique et qui couvre une large période ${ }^{25}$.

Contrairement à ce que l'on pourrait attendre, la brique, commun dénominateur du bâti néerlandais, est le parent pauvre de l'historiographie : l'ouvrage de référence reste la thèse de doctorat de Johanna Hollestelle datant de $1961^{26}$. Un récent colloque en Belgique a néanmoins fait le point sur les recherches consacrées à l'architecture en brique dans le Nord de l'Europe ; on y notera des contributions sur la Hollande et sur la Frise ${ }^{27}$.

Enfin, conforme à la tradition germanique, l'archéologie du bâti aux Pays-Bas ne se limite pas aux grands édifices mais analyse depuis des décennies les maisons urbaines, mettant au jour des structures et des noyaux médiévaux derrière des façades plus récentes. La contribution des archéologues du bâti néerlandais au réseau allemand d'archéologie du bâti domestique (Arbeitskreis für Hausforschung) est importante et régulière ; le dernier volume de la série est d'ailleurs consacré à la Hollande (fig. 3 (28 $^{28}$. Cette Hausforschung systématisée a livré des résultats remarquables à Utrecht, Bois-le-Duc, Amsterdam, Leyde et Maastricht, ainsi que dans des petites villes telles que Zaltbommel et Wijk bij Duurstede ${ }^{29}$. Les archéologues du bâti, pour la plupart indépendants ou rattachés à des administrations urbaines, sont rarement liés au monde académique. Aussi, le travail sur le bâti urbain a-t-il inévitablement un caractère diachronique et local. Isoler le Moyen Âge des autres périodes n'aurait guère de sens dans ce contexte.

\section{Les Pays-Bas médiévaux : la naissance d'une société urbaine}

Certains sujets font l'objet d'une attention particulière, comme la ville, qui pourrait devenir un modèle pour des recherches similaires ailleurs en Europe. À l'exception de quelques villes d'origine romaine (Maastricht, Nimègue, Utrecht) et carolingienne (Deventer, Zutphen), la plupart des villes des Pays-Bas sont nées à partir du XI ${ }^{\mathrm{e}}$ siècle. Le combat permanent contre les " grandes rivières " et la mer, la création de polders de la Zélande et la Hollande jusqu'en Frise et en Groningue ont généré un rapport particulier entre l'homme et son territoire. Né au cœur du Moyen Âge, le "modèle du polder " (poldermodel) est bien plus qu'un réseau de digues et de canaux ; il est une structure sociétale fondée sur des solidarités profondes et vivantes jusqu'à aujourd'hui. L'origine des villes et la vague la fondation de nouvelles cités pendant la seconde moitié du XIV et le début du XV siècle font l'objet de nouvelles études depuis une quinzaine d'années ${ }^{30}$. Cet intérêt pour la ville au Moyen Âge est motivé par les recherches sur les grands développements urbains en Hollande et dans les colonies néerlandaises au XVII ${ }^{e}$ siècle qui s'interrogent sur l'origine des modèles et des villes, idéales ou idéalisées, sur plan régulier. 
Une source exceptionnelle pour les études urbaines est la série de 210 plans de villes, dont 109 des Pays-Bas, levés par le géomètre néerlandais Jacob van Deventer à la demande du roi d'Espagne Philippe II dans les années 1558-1570. Ces plans conservés à Madrid ont fait l'objet d'une remarquable édition en facsimilé, parue entre 1992 et 1998, accompagnée de commentaires topographiques et historiques de C. Koeman, J. C. Visser ${ }^{31}$. Ils présentent les villes des anciens Pays-Bas, à la même échelle, dans leur état à la fin du Moyen Âge, avant les destructions des guerres de religion, la mise en place des nouvelles fortifications à bastions, et les grands développements du XVII ${ }^{\mathrm{e}}$ siècle. Une telle source cartographique n'existe pour aucune autre région en Europe (fig. 4).

Une telle richesse documentaire, liée à l'existence d'archives urbaines généralement conservées à partir du $\mathrm{XV}^{\mathrm{e}}$ siècle, et parfois même plus tôt, permet à des archéologues du bâti et à des historiens de la ville de combiner leurs efforts et de remonter dans le temps, y compris au niveau de la parcelle. La thèse de doctorat de Ad van Drunen consacrée à Bois-le-Duc ('s-Hertogenbosch) est un exemple en la matière ${ }^{32}$. La convergence des sources archéologiques, matérielles et archivistiques lui a permis de remonter jusqu'à la fondation de la ville au XIII ${ }^{\mathrm{e}}$ siècle et de reconstituer l'évolution du parcellaire, du bâti, des propriétaires et des remparts. Résultat de plus de trente ans d'étude, ce travail remarquable a pu s'appuyer sur une politique communale de conservation et de mise en valeur historique ${ }^{33}$. La ville de Bois-leDuc a fait école aux Pays-Bas et pourrait inspirer bien d'autres édiles à travers l'Europe.

Outre la société urbaine, les Pays-Bas médiévaux avaient aussi leur société rurale et féodale.

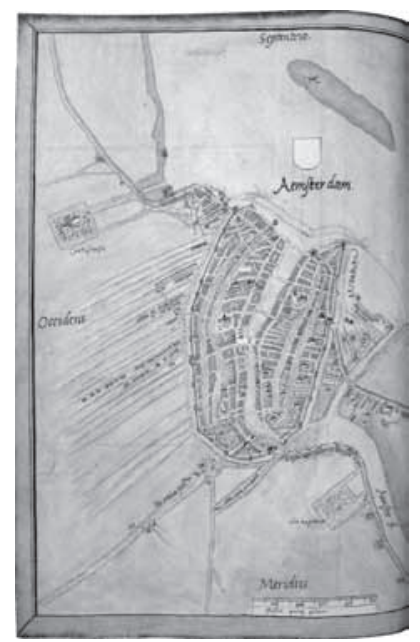

Il n'en reste pas grand-chose, si ce n'est quelques ruines de châteaux forts, une dizaine de châteaux reconstruits au XIX ${ }^{\mathrm{e}}$ siècle et des vestiges mis au jour par des archéologues ${ }^{34}$. La castellologie médiévale néerlandaise est une discipline modeste non seulement par rapport à celle des grands pays voisins, mais aussi par rapport à la prestigieuse époque des résidences de plaisance des XVII ${ }^{\mathrm{e}}$ et XVIII $^{\mathrm{e}}$ siècles aux Pays-Bas ${ }^{35}$. Inversement les études historiques sur la chevalerie et la noblesse médiévale en Hollande, Frise, Zélande, Gueldre, etc. ont connu des développements remarquables ces dix dernières années. Ainsi, par exemple, des historiens ont repéré dans les sources et localisé sur des cartes anciennes plus de cinq cents emplacements de maisons nobles (stinzen) en Frise, alors que les vestiges matériels sont très modestes ${ }^{36}$.

\section{L'édition scientifique : un solide support local}

La recherche en architecture médiévale, comme les autres domaines artistiques, bénéficie de la culture du livre très robuste caractéristique des Pays-Bas. L'édition scientifique n'y est pas le fait des universités ni de l'État, mais des éditeurs privés, parmi lesquels certains ont acquis une carrure internationale de premier plan et publient quasi exclusivement en anglais (Kluwer, Elsevier, Brill, etc.), tandis que d'autres occupent le marché local et publient en néerlandais. Les maisons d'édition commerciales Waanders à Zwolle ou Matrijs à Utrecht accueillent volontiers des monographies d'architecture, tandis que l'éditeur Verloren à Hilversum a fait du Moyen Âge une spécialité et publie la revue $\mathrm{Madoc}^{37}$. L'éditeur Clavis à Utrecht est une fondation non commerciale spécialisée en architecture médiévale. Étant donné la spécificité des sujets et la dimension limitée du marché - en comparaison avec le marché du livre anglophone, germanophone, francophone ou hispanophone -, chaque publication doit trouver un financement propre par le biais de subsides ou de mécénat. Le système est ainsi fait que de nombreuses institutions publiques, notamment les provinces, les communes, le Fonds prince Bernhard, le Fryske Akademy, etc. disposent de budgets spécifiques pour encourager les publications privées.
4. Plan de la ville d'Amsterdam levé par Jacob van Deventer vers 1560 , original conservé à la Biblioteca nacional de España, Madrid, fac-similé photographique dans Canaletto, $1993, n^{\circ} 25$ 


\author{
5. Oude Kerk \\ d'Amsterdam, \\ détails de la \\ Mariakapel, dans \\ Herman Janse, \\ De Oude Kerk \\ te Amsterdam: \\ bouwgeschie- \\ denis en restaura- \\ tie, Zwolle, 2004, \\ p. $142-143$.
}

6. Couverture de la principale revue néerlandaise d'histoire de l'architecture, le Bulletin KNOB, 107/3, 2008.

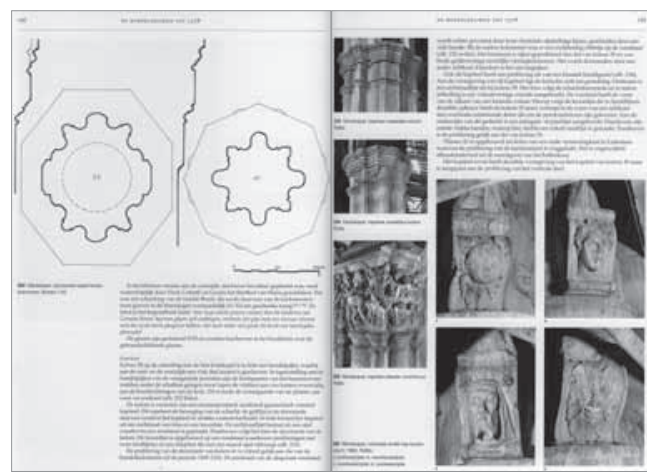

Les dernières monographies publiées en 2003 et 2004 par le service des monuments historiques de l'État, consacrées à la Oude Kerk d'Amsterdam (fig. 5) et à l'église Notre-Dame de Breda, marquent la fin d'un type d'ouvrage exhaustif et érudit dans une série nationale héritée du XIX ${ }^{\mathrm{e}}$ siècle ${ }^{38}$. Le système néerlandais de fondations (stichtingen) qui gèrent, restaurent et financent les édifices, favorise les monographies d'édifices car elles ont un support et un marché local. Des projets individuels, généralement patronnés par la fondation propriétaire de l'édifice en vue d'un jubilé ou d'un autre événement commémoratif, rassemblent des équipes multidisciplinaires qui abordent tous les aspects d'un monument : histoire, archives, fouilles, archéologie du bâti, iconographie, mobilier, orgues, sépultures, restaurations, etc. Moyennant un préfinancement ou un subside local, un des éditeurs précités prend la publication en charge sans grand risque. La qualité de ces ouvrages dépend surtout de la qualité des auteurs. Quoi qu'il en soit, la monographie d'édifice se porte bien mais son contenu ne se limite jamais au seul Moyen Âge ${ }^{39}$. On ne peut pas en dire autant des ouvrages thématiques. Les différentes séries publiées par le service des monuments historiques de l'État ont disparu les unes après les autres ${ }^{40}$. Les ouvrages thématiques sont plus rares et sont le fait d'initiatives isolées, comme celui sur les remplages gothiques publié en 1998 ou celui sur le développement historique des châssis de fenêtre paru en $2010^{41}$. Il est exceptionnel qu'un musée consacre une exposition à l'architecture médiévale ; celle organisée en 2002 par Den Hartog sur la sculpture architectonique romane à Maastricht fut en soi un événement ${ }^{42}$.
Si le Bulletin $K N O B$, déjà cité, est la seule revue néerlandaise qui publie sur des sujets d'architecture, il existe quantité de revues locales dédiées au patrimoine (fig. 6). Les Néerlandais ne rechignent pas à s'impliquer personnellement en devenant membre de fondations privées ou en s'engageant dans la vie associative patrimoniale et muséale. Ainsi, par exemple, toutes les fondations qui gèrent des églises historiques, médiévales pour la plupart, publient pour leurs membres des revues qui se font l'écho des dernières découvertes, des restaurations, des éditions d'archives, des activités, etc. Des fondations telles que Oude Groninger Kerken (Groningue, fondée en 1969), Alde Fryske Tjerken (Frise, 1970), Oude Hollandse Kerken (Hollande, 1975), Oude Gelderse Kerken (Gueldre, 1975), Oude Zeeuwse Kerken (Zélande, 1976), Oude Drentse Kerken (Drenthe, 2003), etc. jouent un rôle primordial dans la conservation d'édifices anciens, très importante dans un pays où le processus de désaffectation des lieux de culte est particulièrement avancé.

La langue reste néanmoins un obstacle relatif à la diffusion internationale des résultats de la recherche. C'est principalement par des articles publiés dans des revues scientifiques internationales que les universitaires et les chercheurs néerlandais atteignent la communauté scientifique internationale. Le marché du livre sur des sujets liés aux Pays-Bas reste, quant à lui, essentiellement néerlandais. Il a déjà été signalé que les archéologues du bâti néerlandais publient volontiers en allemand, notamment par le biais du réseau Arbeitskreis für Hausforschung. L'ouvrage majeur sur le financement de la construction des cathédrales au Moyen Âge publié par Wim Vroom en 1981 vient seulement de paraître en anglais en 2010,

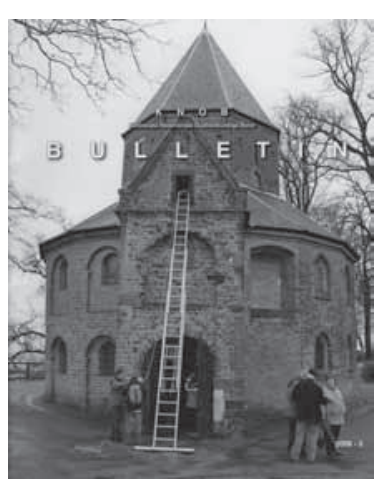
dans une édition augmentée ${ }^{43}$. Dans les vingt dernières années, seuls deux ouvrages sont parus en français sur des aspects de l'architecture médiévale aux Pays-Bas, aux éditions Zodiaque et chez Citadelles $\delta$ Mazenod $^{44}$. 


\section{Architecture médiévale et patrimoine : recherche et autres défis}

La question des relations entre l'université et le monde de la conservation du patrimoine ne se limite évidemment pas à l'architecture médiévale, mais concerne le patrimoine dans sa totalité et sa diversité. Les relations avec l'expertise et la connaissance universitaire ont longtemps été le fondement de la politique patrimoniale, y compris au plan théorique et international, comme le prouvent notamment les ouvrages de Wim F. Denslagen ${ }^{45}$. Aujourd'hui, les deux secteurs sont frappés d'une crise profonde, identitaire et économique, et il est évident que les modèles qui prévalaient jusqu'au début de ce siècle ont vécu. Si la notion de patrimoine s'est considérablement étendue, la gestion de ce dernier se réduit de plus en plus à des questions de management qui s'embarrassent moins de la recherche fondamentale que de considérations économiques, touristiques et politico-identitaires. Cette approche de "bon gestionnaire " est particulièrement constitutive de l'identité néerlandaise, tant au niveau de l'État que des communes qui, aux Pays-Bas, ont un rôle très important en matière patrimoniale (certaines comme Utrecht et Bois-le-Duc sont vraiment exemplaires). Il est d'ailleurs révélateur que le service des monuments de l'État, initialement appelé le Rijksdienst voor de Monumentenzorg (RDMZ), a été restructuré et a changé de nom à deux reprises durant la dernière décennie, pour devenir au final le Rijksdienst voor het Cultureel Erfgoed (RCE).

La recherche universitaire est également en crise et subit les coupes budgétaires du gouvernement. Des projets de recherche fondamentale sur des sujets d'architecture médiévale ont peu de chance d'être retenus car leur " pertinence sociétale " sera jugée insuffisante. L'université est donc condamnée à innover en développant une approche patrimoniale fondée sur des synergies entre sciences humaines et sciences exactes, et des problématiques en phase avec les besoins sociaux, le développement durable, etc. Le cas de la désaffectation des églises, y compris des églises médiévales classées, est un problème particulièrement aigu aux Pays-Bas. L'approche scientifique de ces bâtiments n'est plus confinée à l'expertise de l'archéologue, de l'historien de l'art et de l'architecte, mais implique un faisceau de disciplines complémentaires telles que le droit, l'économie, la sociologie, et même l'éthique ${ }^{46}$.

En matière de recherche universitaire, les Pays-Bas ont traditionnellement développé des programmes ambitieux, internationaux et innovants, mais limités dans le temps. Le rendement immédiat n'est pas favorable au développement de pôles d'excellence à long terme, et l'innovation à tout prix n'est guère compatible avec le travail de fond au niveau local. Cela donne lieu à des divorces profonds entre d'une part des professeurs d'université et leurs équipes travaillant sur des sujets internationaux et publiant en anglais dans les revues scientifiques de niveau Al, comme le leur demandent leurs universités, et d'autre part, les acteurs du terrain et les éditeurs locaux, les restaurateurs et les archéologues du bâti. L'un deux, Karel Emmens, affirmait récemment : «Depuis l'éméritat de Aart Mekking il y a quelques années, il n'y a plus de stimulant à la recherche, les collaborations internationales se réduisent, et l'échange de connaissances se tarit. Malgré les divergences d'opinion, sa chaire offrait une plateforme de recherche pour l'architecture médiévale. Espérons que cette carence pourra être comblée rapidement $"{ }^{47}$. Gageons que le nouveau Center for Medieval Studies Amsterdam, inauguré ce 14 septembre 2011 à l'université d'Amsterdam (CMSA), ainsi que le professeur Lex Bosman, médiéviste et nouveau titulaire de la chaire d'histoire de l'architecture à cette université, relèvent le défi avec succès.

1. Koos Bosma et al. éd., Bouwen in Nederland 600-2000, Amsterdam/Zwolle, 2007. La partie sur le Moyen Âge, coordonnée par Aart Mekking, couvre les p. 12-229.

2. Frans A. J. Vermeulen, Handboek tot de geschiedenis der Nederlandsche bouwkunst, 4 vol., La Haye, 1928-1941 ; S. J. Fokkema Andreae, R. C. Hekker, E. H. ter Kuile, Duizend jaar bouwen in Nederland, 2 vol., Amsterdam, 1957-1958.

3. Willem Pieter Blockmans, Walter Prevenier, The Promised Lands: The Low Countries under Burgundian Rule, 1369-1530, Philadelphie, 1997 ; Wim Blockmans, Metropolen aan de Noordzee: De geschiedenis van Nederland, 1100-1560, Amsterdam, 2010.

4. Le Contactgroep Signum, par exemple, travaille sur l'Église médiévale aux Pays-Bas : www.contactgroepsignum.eu. 
5. Aart J. J. Mekking, De Sint-Servaaskerk te Maastricht: Bijdragen tot de kennis van de symboliek en de geschiedenis van de bouwdelen en de bouwsculptuur tot ca. 1200, Utrecht, 1986 ; Alex F. W. Bosman, De Onze Lieve Vrouwekerk te Maastricht: bouwgeschiedenis en historische betekenis van de oostpartij, Utrecht, 1990 ; Elizabeth den Hartog, Romanesque Architecture and Sculpture in the Meuse Valley, Leeuwarden/Malines, 1992.

6. Günter Bandmann, Mittelalterliche Architektur als Bedeutungsträger, Berlin, 1951 (éd. angl. : Günter Bandmann, Early Medieval Architecture as Bearer of Meaning, New York, 2005) ; Richard Krautheimer, "Introduction to an 'Iconography of medieval Architecture' ", dans Journal of the Warburg and Courtauld Institutes, 5, 1942, p. 1-33.

7. Voir notamment Aart J. J. Mekking, Het spel met toren en kapel: Bouwen pro en contra Bourgondië van Groningen tot Maastricht, Utrecht/Zutphen, 1992, et la réaction à cet ouvrage : Kees van der Ploeg, "Wat kan architectuur in de middeleeuwen betekenen? ", dans Bulletin KNOB, 91/3-4, 1992, p. 121-127.

8. Jeroen Westerman, "Art and Region ", dans Kunstchronik, 51/2, 1998, p. 49-51.

9. Leonhard Helten, Kathedralen für Bürger: Die St. Nikolauskirche in Kampen und der Wandel architektonischer Leitbilder städtischer Repräsentation im 14. Jahrhundert, Utrecht, 1994 ; Jos Stöver, De Salvator-ofOudmunsterkerkte Utrecht:stichtingsmonument van het bisdom Utrecht, Utrecht, 1997 ; Klaus Hardering, Die Abteikirche von Klosterrath: Baugeschichte und Bedeutung/De Abdijkerk te Rolduc: bouwgeschiedenis en betekenis, Utrecht, 1998 ; Herman J. Lenferink, De Plechelmuskerk te Oldenzaal: vorm, geschiedenis en betekenis, Utrecht, 1998 ; Jeroen Westerman, " Notre-Dame de Tournai au XII ${ }^{\mathrm{e}}$ siècle. Une cathédrale 'revendicatrice' ", dans Jacqueline Hamesse éd., Bilans et perspectives des études médiévales (1993-1998), (colloque, Barcelone, 1999), Louvain-la-Neuve, p. 359374 ; Thomas Coomans, "L'architecture médiévale des ordres mendiants (Franciscains, Dominicains, Carmes et Augustins) en Belgique et aux Pays-Bas ", dans Revue belge d'archéologie et d'histoire de l'art, 70, 2001, p. 3-111 ; Thomas Coomans, "Cistercian Nunneries in the Low Countries: The Medieval Architectural Remains ", dans Meredith Parsons Lillich éd., Studies in Cistercian Art and Architecture 6. (Cistercian Studies Series, 194), Kalamazoo, 2005, p. 61131 ; Emanuel Klinkenberg, Compressed Meanings: The Donor's Model in Medieval Art to around 1300, (Architectura medii aevi, 2), Turnhout, 2009 ; Emanuel Klinkenberg, Architectuuruitbeelding in de Middeleeuwen: oorsprong, verbreiding en betekenis van architectonische beeldtradities in de WestEuropese kunst tot omstreeks 1300, Utrecht, 2010 ; Uta Maria Bräuer, Emanuel S. Klinkenberg, Jeroen Westerman éd., Kunst $\theta$ Region: Architektur und Kunst im Mittelalter, Beiträge einer Forschungsgruppe/Art $\theta$ Region: Architecture and Art in the Middle Ages, Contributions of a Research Group, Utrecht, 2005 ; Elizabeth den Hartog et al. éd., Bouwen en duiden: studies over architectuur en iconologie, Alphen aan de Rijn, 1994.

10. Elizabeth den Hartog, De oudste kerken in Holland: van kerstening tot 1300, Utrecht, 2002 ; Elizabeth den Hartog, Romanesque Sculpture in Maastricht, Maastricht, 2002.

11. Le poids des anciennes études apparaît clairement dans Jan Esther, "Le Brabant ", dans Marjan Buyle et al.,
L'architecture gothique en Belgique, Bruxelles, 1997, p. 82103. Sur l'historiographie de "l'école brabançonne", voir Thomas Coomans, "'Brabantse gotiek' of 'Gotiek in Brabant'? Ontstaan van een architectuurschool, status quaestionis en onderzoeksperspectieven ", dans La Ville Brabançonne/De Brabantse Stad, (colloque, Louvain, 2002), numéro thématique de Bijdragen tot de Geschiedenis, 86/3-4, 2003, p. 241-271.

12. Krista De Jonge, Piet Geleyns, Markus Hörsch, Gotiek in het hertogdom Brabant, Louvain, 2009.

13. Merlijn Hurx, De particuliere bouwmarkt in de Nederlanden en de opkomst van architect (1350-1530), thèse, université de Delft, 2010 (publication en anglais en cours).

14. Thomas Coomans, "L'apogée du gothique dans les villes des anciens Pays-Bas avant la Réforme (1492-1530) ", dans Monique Chatenet et al. éd., Le Gothique de la Renaissance, $I V$ rencontres d'architecture européenne, (colloque, Paris, 2007), (De Architectura, 13), Paris, 2011, p. 65-87.

15. Justin Kroesen, Regnerus Steensma, The Interior of the Medieval Village Church/Het middeleeuwse dorpskerkinterieur, Louvain, 2004.

16. Sur le fameux jubé d'Amersfoort, voir Karel Emmens, "Het doxaal in de Sint-Joriskerk te Amersfoort", dans Bulletin KNOB, 94/3-4, 1995, p. 81-90.

17. On compte, parmi les découvertes majeures, les peintures de l'église de Britsum (Frise) du début du XIII ${ }^{\mathrm{e}}$ siècle, publiées dans un numéro thématique du Bulletin KNOB, 99/1-2, 2000, p. 1-52.

18. Christel Theunissen, Jos Koldeweij, De koorbanken van Oirschot en Aarschot: gezien door de lens van Hans Sibbelee en Jan Verspaandonk, Nimègue, 2011.

19. Le dernier en date : Ronald Stenvert, Gabri van Tussenbroek éd., Inleiding in de bouwhistorie: opmeten en onderzoeken van oude gebouwen, Utrecht, 2007.

20. Les suivants sont des exemples d'excellentes analyses du bâti de monuments médiévaux : Barbara Perlich, Gabri van Tussenbroek, «De Valkenhofkapel te Nijmegen. Nieuwe gegevens over de middeleeuwse bouwgeschiedenis ", dans Bulletin KNOB, 107, 2008, p. 90-100 ; Karel Emmens, "Een nieuwe bouwgeschiedenis voor de Nieuwstadskerk te Zutphen ", dans Bulletin KNOB, 103, 2004, p. 122-137 ; Hein Hundertmark, "De lappenkerk van Limmen ", dans Bulletin KNOB, 104, 2005, p. 37-57.

21. Herman Janse, Dirk Jan de Vries, Merk en werk van de steenhouwer: het steenhouwersambacht in de Nederlanden voor 1800, Zwolle/Zeist, 1991.

22. Wim Dubelaar et al. éd., Utrecht in steen: historische bouwstenen in de binnenstad, Utrecht, 2007.

23. Gabri van Tussenbroek, The Architectural Network of the Van Neurenberg Family in the Low Countries (1480-1640), (Architectura moderna, 4), Turnhout, 2006 ; Hurx, 2010, cité n. 13.

24. Voir notamment Dirk Jan de Vries, Bouwen in de late middeleeuwen: stedelijke architectuur in het voormalige Over- en Nedersticht, Utrecht, 1994 ; Dirk Jan de Vries éd., Monumenten en bouwhistorie: jaarboek Monumentenzorg 1996, Zwolle/Zeist, 1996 ; et le numéro thématique sur la dendochronologie de Bulletin KNOB, 99/3, 2000, p. 61-94. 
25. Herman Janse, Houten kappen in Nederland (1000-1940), Delft, 1989.

26. Johanna Hollestelle, De steenbakkerij in de Nederlanden tot omstreeks 1650, (thèse, université d'Utrecht, 1961), Arnhem, 1974.

27. Karel Emmens, «De oudste Friese baksteen. Een heroriëntatie op de introductie en vroege toepassingen van baksteen in Friesland en Groningen », et Gabri van Tussenbroek, "Vroege baksteen in Holland tot 1300 ", dans Thomas Coomans, Harry van Royen éd., Medieval Brick Architecture in Flanders and Northern Europe: The Question of the Cistercian Origin, (Novi monasterii, 7), Koksijde, 2007, p. 73-114 et 115-132.

28. Gabri van Tussenbroek éd., Hausbau in Holland: Baugeschichte und Stadtentwicklung, (Jahrbuch für Hausforschung, 61), Marbourg, 2010. Voir également Hausbau in den Niederlanden/Bouwstenen voor oude woonhuizen in Nederland, (Jahrbuch für Hausforschung, 39), Marbourg, 1990.

29. Gabri van Tussenbroek, Onder de daken van Zaltbommel: bouwen en wonen in de historische binnenstad (1350-1650), Utrecht, 2003 ; M. A. van der Eerden-Vonck, J. Hauer, G. W. J. van Omme, Wijk bij Duurstede 700 jaar stad: ruimtelijke structuur en bouwgeschiedenis, Hilversum, 2000.

30. Particulièrement intéressants sont le numéro thématique dirigé par Wim Boerefijn, du Bulletin KNOB, 102, 2003, p. 121-137, et Wim Boerefijn, The Foundation, Planning and Buildings of New Towns in the $13^{\text {th }}$ and $14^{\text {th }}$ Centuries in Europe, thèse, université d'Amsterdam, 2010.

31. C. Koeman, J. C. Visser éd., De stadsplattegronden van Jacob van Deventer: facsimile's van de minute en netkaarten van de steden in het huidige Nederland, Duitsland en Luxemburg vervaardigd door Jacob van Deventer tussen 1558 en 1570, 8 vol., Alphen aan de Rijn, 1992-1998.

32. Ad van Drunen, 's-Hertogenbosch van straet tot stroom, Zwolle, 2006.

33. Hans Willems et al., De onderste steen boven: 25 jaar bouwhistorie in 's-Hertogenbosch, Utrecht, 2000.

34. Sur les vestiges de châteaux aux Pays-Bas, voir Hans L. Janssen, J. M. M. Kylstra-Wielinga, Ben Olde Meierink, 1000 jaar kastelen in Nederland: Functie en vorm door de eeuwen heen, Utrecht, 1996, ainsi que le numéro thématique sous la direction de Hans L. Janssen, Bulletin KNOB, 109, 2/3, 2010, p. 49-104.

35. Pour plus d'information sur la fondation des châteaux néerlandais (Nederlandse Kastelenstichting), consulter leur site : www.kastelen.nl.

36. P. N. Noomen, De stinzen in middeleeuws Friesland en hun bewoners, Hilversum, 2009.

37. La revue trimestrielle Madoc, Tijdschrift over de Middeleeuwen existe depuis 1986. L'architecture médiévale est quasi absente de la revue médiéviste Millennium, tijdschrift voor middeleeuwse studies, fondée en 1987.

38. Dans la fameuse série De Nederlandse monumenten van geschiedenis en kunst, voir Gerard van Wezel, De OnzeLieve-Vrouwekerk en Grafkapel voor Oranje-Nassau te Breda, Zwolle, 2003, et Herman Janse, De Oude Kerk te Amsterdam: bouwgeschiedenis en restauratie, Zwolle, 2004.
39. La dernière en date est Elizabeth den Hartog, John Veerman éd., De Pieterskerk in Leiden: Bouwgeschiedenis, interieur en gedenktekens, Zwolle, 2011.

40. Signalons notamment Jaarboek monumentenzorg, série d'ouvrages thématiques annuels sur la conservation du patrimoine.

41. H. J. Tolboom, Venstertraceringen in Nederland, La Haye, 1998 ; Jan Jehee, Tussen lucht en licht: de ontwikkeling van de vensters, kozijnen ramen en luiken, 2010.

42. De weg naar het Paradijs, Elizabeth den Hartog éd., (cat. expo., Maastricht, Bonnenfanten Museum, 2003-2004), Maastricht, 2003, et Elizabeth den Hartog, Romanesque Sculpture in Maastricht, Maastricht, 2002.

43. Wim Vroom, Financing the Cathedral Building in the Middle Ages: The Generosity of the Faithful, Amsterdam, 2010.

44. Ada van Deijk, Pays-Bas romans, La-Pierre-qui-Vire, 1994 [éd. orig. : Romaans Nederland, Amsterdam, 1994] ; Thomas Coomans, "L'architecture", dans Christian Heck éd., L'Art flamand et hollandais : le siècle des Primitifs, 1380-1520, (L'art et les grandes civilisations, 33), Paris, 2003, p. 7-120 et 541-591.

45. Wim F. Denslagen, Architectural Restoration in Western Europe: Controversy and Continuity, Amsterdam, 1994 [éd. orig. : Omstreden herstel: Kritiek op het restaureren van monumenten: een thema uit de architectuurgeschiedenis van Engeland, Frankrijk, Duitsland en Nederland (1779-1953), La Haye, 1987].

46. 2008 fut déclarée "année du patrimoine religieux " aux Pays-Bas et déboucha sur des recommandations, présentées dans Nico Nelissen, Geloof in de toekomst! Strategisch Plan voor het religieus erfgoed, Utrecht, 2008.

47. «Sinds Aart Mekking enkele jaren geleden met emeritaat is gegaan, is er geen aanjager meer van onderzoek, verdwijnen internationale contacten en samenwerking, en gaat kennisuitwisseling verloren. Hoezeer inzichten ook konden verschillen, diens hoogleraarschap bood een platform voor onderzoek naar middeleeuwse architectuur. Het is te hopen dat in deze leemte op korte termijn opnieuw zal worden voorzien" (Karel Emmens, chronique dans Bulletin KNOB, 109/2-3, 2010, p. 106).

Thomas Coomans, Katholieke Universiteit Leuven thomas.coomans@asro.kuleuven.be

Jeroen Westerman, Gelders Genootschap, Arnhem/Universiteit Leiden

j.westerman@planet.nl

Mots-clés : archéologie du bâti, architecture et urbanisme, Moyen Âge, patrimoine, statut disciplinaire, transdisciplinarité. 